\title{
Magnetic field effects on pineal indoleamine metabolism and possible biological consequences
}

\author{
RUSSEL J. REITER ${ }^{1}$ AND BRUCE A. RICHARDSON*
}

Department of Cellular and Structural Biology, The University of Texas Health Science Center at San Antonio, San Antonio, Texas 78284, USA; and *Department of Biology, The University of Michigan-Flint, Flint, Michigan 48502, USA

ABSTRACT In recent years, there has been a great deal of publicity concerning the possible health effects of electric and/or magnetic field exposure. One of the most frequently reported observations after the exposure of animals to either electric or magnetic fields relates to alterations in the metabolism of serotonin (5HT) to melatonin within the pineal gland. This review summarizes these results particularly in animals exposed to intermittently inverted, non-time varying magnetic fields, i.e., pulsed static magnetic fields. When exposure occurs at night, the converstion of 5HT to melatonin is typically depressed, not unlike that after light exposure at night. The mechanisms by which pulsed magnetic fields alter the ability of the pineal to convert $5 \mathrm{HT}$ to the chief pineal hormone melatonin remains unknown but may involve effects on any or all of the following: the retinas, the suprachiasmatic nuclei, the peripheral sympathetic nervous system, and the pinealocytes. Results to date suggest that induced electrical currents (eddy currents) produced by the pulsed magnetic fields are particularly detrimental to pineal indoleamine metabolism and may be an important causative factor in the metabolic changes measured. The physiological consequences of perturbations in the melatonin rhythm induced by magnetic field exposure remain unknown. — Reiter, R. J.; Richardson, B. A. Magnetic field effects on pineal indoleamine metabolism and possible biological consequences. FASEB J. 6: 2283-2287; 1992.

Key Words: pineal gland - melatonin - static magnetic fields • serotonin - metabolism

BECAUSE OF THE VIRTUAL UBIQUiTY of electrical appliances and apparatus in modern society, animals (including humans) live in an extremely complex electric and magnetic field environment. Studies during the last decade suggest that both electric and magnetic fields, either alone or in combination, may induce biological effects in organisms that could have untoward consequences, even though the fields are generally considered to be innocuous. The purpose of the current report is to summarize the physiologic changes induced by what is referred to as pulsed, static magnetic fields (and the resulting induced electric currents or eddy currents) in a portion of the brain referred to as the pineal gland. Pulsed static magnetic fields describe direct current magnetic fields that are intermittently changed (pulsed) in some way. In the present review, we discuss primarily the inversion of the horizontal component of the Earth's magnetic field (the geomagnetic field) and the resulting changes in pineal melatonin production. Pineal gland metabolism was selected as a focus of discussion in this report because it is one of the endpoints that is most consistently perturbed by alterations in the electromagnetic environment (1-3).

\section{THE PINEAL AND ITS HORMONE MELATONIN}

The pineal gland in mammals is an end organ of the visual system. The gland is connected to the eyes by a series of neurons which involve: 1) retinohypothalamic fibers that synapse in the suprachiasmatic nuclei (SCN) ${ }^{2}$ of the hypothalamus, 2) projections from the SCN to the area of the paraventricular nuclei (PVN), 3) long descending fibers from the PVN region that synapse on preganglionic sympathetic cell bodies in the intermediolateral cell column of the upper thoracic cord, 4) peripheral processes of these cells that leave the central nervous system to synapse in the superior cervical ganglia, and 5) peripheral postganglionic sympathetic neurons that terminate within the pineal gland (Fig. 1) (4). In the pineal, the postganglionic sympathetic fibers end in the vicinity of pinealocytes, the hormone-producing cells of the pineal gland; at night the postganglionic sympathetic neurons release a catecholaminergic neurotransmitter, norepinephrine (NE), which then interacts with membrane-bound receptors (both $\beta$ - and $\alpha$-adrenergic receptors) in the pinealocyte membrane (5). This interaction, via the intracellularly produced second-messenger cyclic adenosine monophosphate (cAMP), induces a rise in the synthesis and secretion of the chief pineal hormone melatonin. The generation of melatonin production at night involves an increase in the activity of the enzyme, $\mathrm{N}$-acetyltransferase (NAT), which rate limits melatonin production (Fig. 2). NAT acts on serotonin (5-hydroxytryptamine or $5 \mathrm{HT}$ ) to convert it to $\mathrm{N}$-acetylserotonin (NAS); NAS is subsequently synthesized to melatonin in the presence of the enzyme hydroxyindole- $\mathrm{O}$ methyltransferase (HIOMT) (6). The high levels of melatonin produced at night in the pineal gland are quickly released into the blood, and as a result blood levels of melatonin measured at night are typically higher than those measured during the day (7). This day/night rhythm of

\footnotetext{
'To whom correspondence should be addressed, at: Department of Cellular and Structural Biology, The University of Texas Health Science Center at San Antonio, 7703 Floyd Curl Dr., San Antonio, Texas 78284, USA.

${ }^{2}$ Abbreviations: SCN, suprachiasmatic nuclei; PVN, paraventricular nuclei; NE, norepinephrine; 5HT, serotonin; 5HIAA, 5-hydroxyindole acetic acid; TRP, tryptophan; MF, magnetic field; $\mu \mathrm{T}$, micróTesla; NAT, N-acetyltransferase; NAS, N-acetylserotonin; cAMP, cyclic adenosine monophosphate; 50HTRP, 5-hydroxytryptophan.
} 


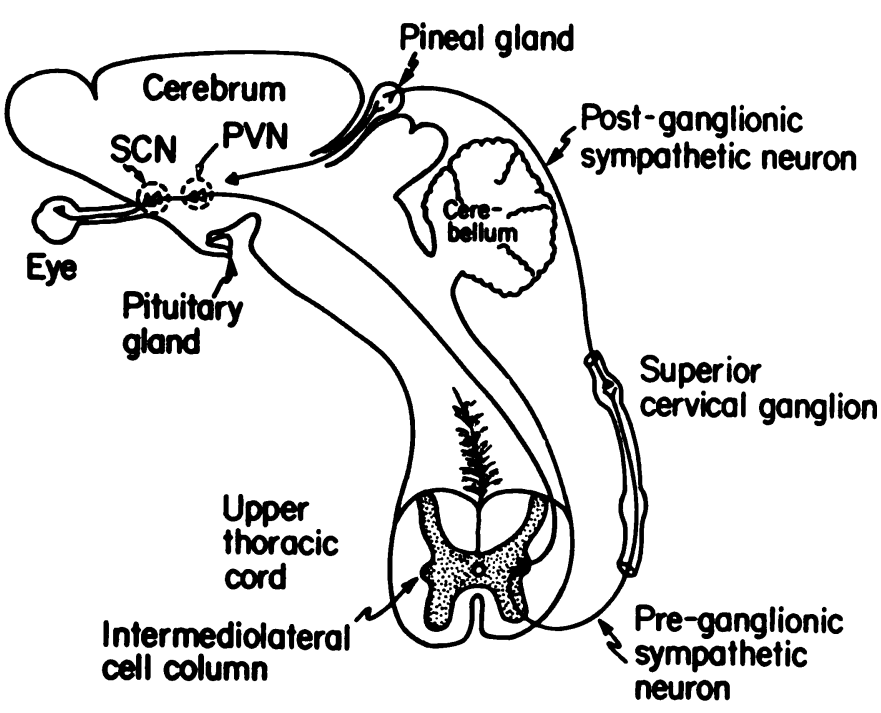

Figure 1. Neural pathways that connect the eyes to the pineal gland in mammals, including humans. The fibers connecting the eyes to the suprachiasmatic nuclei (SCN) of the hypothalamus are referred to as the retinohypothalamic tract. Besides fibers in the central nervous system, neurons in the peripheral nervous system (i.e., preand postganglionic sympathetic fibers) also participate in relaying light/dark information from the eyes to the pineal gland. PVN, paraventricular nuclei. From ref 4).

melatonin production provides important timing information to the body and is necessary for optimal function of the organism.

5HT, besides being converted to melatonin, is also metabolized via another pathway to the metabolite 5-hydroxyindole acetic acid (5HIAA) (Fig. 2). This compound serves as a precursor (Fig. 2) for other substances produced in the pineal gland, whose function in terms of organismal physiology remains unidentified. $5 \mathrm{HT}$ is synthesized from an amino acid tryptophan (TRP) taken into the pinealocyte from the blood. The conversion of TRP to 5HT involves an intermediate compound 5-hydroxytryptophan (50HTRP).

\section{MAGNETIC FIELD PERTURBATIONS AND ALTERATIONS IN PINEAL METABOLISM}

According to Welker and colleagues (8), the exposure of rats to an alternation of the Earth's static magnetic field (MF) [field strength, approximately 0.5 Gauss or 50 microTesla $(\mu \mathrm{T})]$ significantly depressed the activity of the rate-limiting enzyme in melatonin production, i.e., NAT and the levels of melatonin itself in the pineal gland. Furthermore, this group reported that merely a $5^{\circ}$ horizontal rotation of the Earth's MF was able to change pineal indoleamine metabolism; because of this they concluded that the gland was directly "magnetosensitive." The induction of pineal metabolic and physiologic changes by weak, static MF have also been reported by other authors (9-13) with the bulk of the evidence suggesting that MF exposure, by mechanisms that could be either direct or indirect, changes both the electrical activity of the pinealocytes or their ability to produce the critical pineal hormone melatonin. A recent study also indicates that production of the intracellular second messenger, i.e., cAMP, which mediates the nocturnal rise in pineal NAT activity and melatonin content, is depressed by the exposure of rats to static MF (12).

In an attempt to further define the relationships of static MF exposure on pineal serotonin metabolism, Lerchl and co-workers $(14,15)$ carried out a series of studies of rodents during the night when pineal melatonin synthesis is naturally elevated. The following experimental paradigm was used in these studies. A pair of Helmholtz coils $(1 \mathrm{~m}$ diameter with a clearance of $0.5 \mathrm{~m}$ ) was oriented in a north/south direction. The measured strength of the horizontal component of the geomagnetic field was 0.4 Gauss $(40 \mu \mathrm{T})$. The coils produced a rapid inversion of the horizontal component when connected by a relay switch to a DC power supply at approximately 12 volts and the appropriate current (Fig. 3). Using this apparatus, the reversal of MF could be accomplished within approximately $25 \mathrm{~ms}$, while its compensation was reached at about $5 \mathrm{~ms}$ after the coils were activated (Fig. 4). On the night of the study, beginning $3.5 \mathrm{~h}$ after onset of darkness, young adult rats or mice were placed between the coils and exposed to an altered geomagnetic field for $1 \mathrm{~h}$; during this interval the coils were automatically (and instantaneously) activated or deactivated 6 times each at regular intervals of $5 \mathrm{~min}$. A the end of the $1 \mathrm{~h}$ exposure period, the animals were decapitated and the pineal glands were collected for biochemical analyses. All nighttime exposure procedures were carried out under a weak red light that has no effect on pineal melatonin production (16).

As a consequence of the $1 \mathrm{~h}$ exposure to the repeatedly pulsed static MF, the pineal gland of both male rats and male and female mice (Fig. 5) exhibited a significant increase in 5HT levels. Furthermore, 5HIAA values were likewise elevated in the pineal gland whereas activity of the rate-limiting enzyme in melatonin production, i.e., NAT, was depressed. It was presumed that the rise in pineal $5 \mathrm{HT}$ was a direct con-

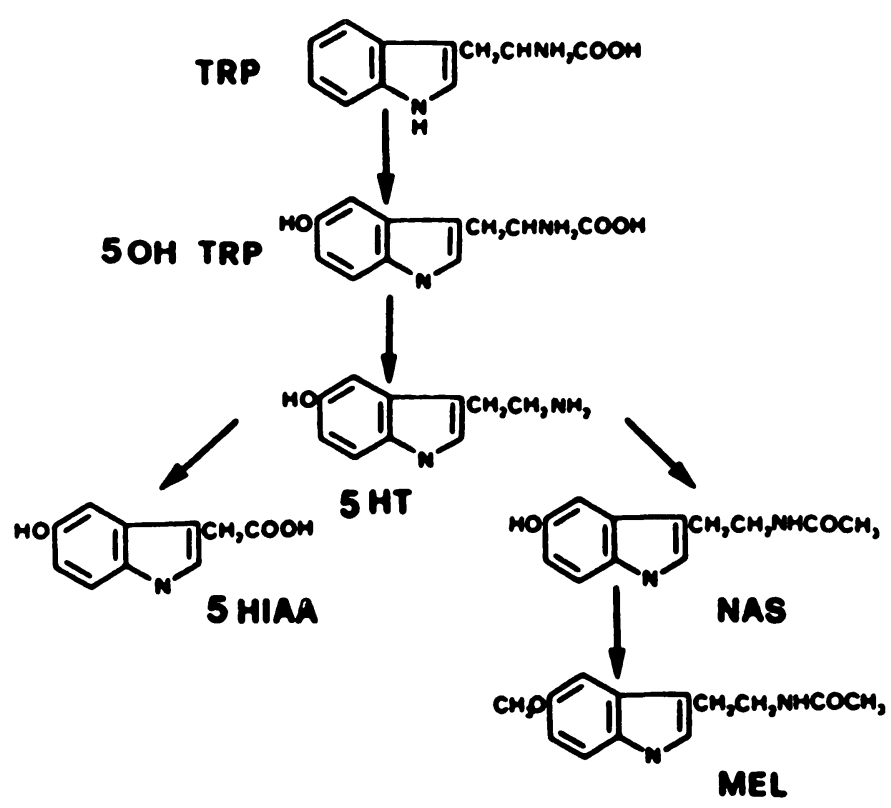

Figure 2. Pathway for the production of melatonin (MEL), an important pineal hormone, from the amino acid tryptophan (TRP) via serotonin (5HT). The conversion of $5 \mathrm{HT}$ to melatonin involves two enzymes, $\mathrm{N}$-acetyltransferase or NAT, which converts $5 \mathrm{HT}$ to $\mathrm{N}$-acetylserotonin (NAS) and hydroxyindole-O-methyltransferase or HIOMT, which converts NAS to melatonin. The activity of the enzyme NAT increases at night in the pineal gland and determines the amount of melatonin produced; as a result, pineal and blood levels of melatonin also increase at night. Besides being metabolized to melatonin, 5HT can be converted to 5-hydroxyindole acetic acid (5HIAA) by the enzyme monoamine oxidase (MAO). The intermediate factor in the conversion of TRP to 5HT is 5-hydroxytryptophan (5OHTRP). 


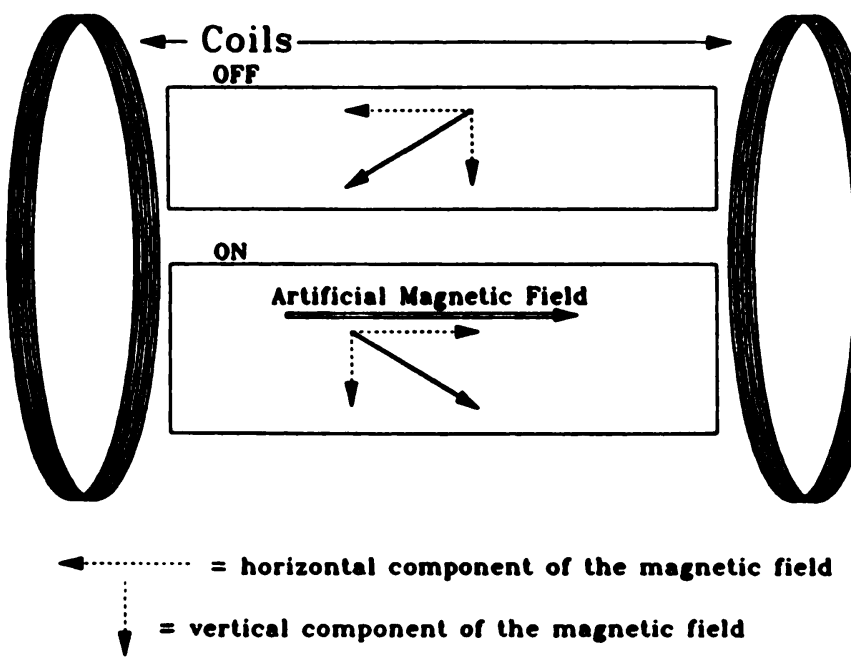

Figure 3. Diagrammatic representation of the inversion of the geomagnetic field in the experiments performed by Lerchl and colleagues $(14,15)$. The solid arrow between the two dashed arrows represents the geomagnetic field vector under normal circumstances (off condition) and after its inversion (on condition).

sequence of the reduction in $5 \mathrm{HT}$ acetylation by NAT; likewise, as $5 \mathrm{HT}$ accumulated it was quickly oxidatively deaminated to 5HIAA. Typically, the concentrations of 5HT and melatonin in the pineal gland are inversely related; thus, in the presence of high NAT activity, melatonin is formed at the expense of 5HT. In contrast, when NAT activity is suppressed, e.g., by $\mathrm{MF}$ perturbations, $5 \mathrm{HT}$ accumulates whereas melatonin values drop. Rises in pineal $5 \mathrm{HT}$ and 5HIAA were observed in the studies reviewed previously. Considering the high levels of 5HT and 5HIAA in the pineal gland (17), the increases observed could be regarded as a major physiological consequence of alterations in the geomagnetic field.

Lerchl et al. (15) repeated the initial study and extended the experiment by including rats that were also exposed to inverted MF but in which the application of the MF was changed in an important way. Coils identical to those used in the initial report were again used. At $3.5 \mathrm{~h}$ after onset of darkness, one group of rats was subjected to the automatic inversion (by means of a relay switch) of the geomagnetic field at 1-min intervals for $1 \mathrm{~h}$. A second group of rats was also exposed to the same frequency of MF inversion; however, in this case the voltage was regulated manually by means of an integrating potentiometer. The manual procedure required about $1 \mathrm{~s}$ for activating or deactivating the artificial MF, whereas with the use of the relay switch the voltage in the coils changes almost instantaneously. In both cases, the artificially generated field inverted the horizontal component of the Earth's MF; however, the inductivity of the coils allowed for the production of a measurable induced electrical current (eddy current) only in those animals exposed to the automatically inverted fields (Fig. 4). The rate of change of the generated MF ( $\mathrm{dB} / \mathrm{dt})$ was estimated by measuring the eddy currents induced in a search coil placed between the Helmholtz coils. Whereas the automatic activation of the Helmholtz coils produced eddy currents when the power supply was switched on and off (maximal $\mathrm{dB} / \mathrm{dt}$ values occurring at the moments of connecting and disconnecting the voltage), manual activation and deactivation of the Helmholtz coils (during a $1 \mathrm{~s}$ interval) with the integrating potentiometer did not produce measurable eddy currents in the search coil (15).
These two means of activation of the Helmholtz coils with inversion of the MF produced very different effects on pineal 5HT metabolism. As in the initial study (14), in rats exposed to the automatically inverted fields (with induced eddy current) pineal 5HT and 5HIAA levels were elevated $1 \mathrm{~h}$ later. Likewise, this treatment significantly depressed pineal NAT activity and melatonin levels (Fig. 4). In contrast, in rats exposed to the manually inverted fields (with little or no induced eddy currents) pineal 5HT, 5HIAA, and melatonin levels as well as the activity of the rate-limiting enzyme in melatonin production, NAT, remained unaltered (15).

Although these studies clearly indicate that indoleamine metabolism in the pineal gland is altered by MF changes during the night, the pineal gland may not be the only organ in which neurochemical changes occur as a result of such treatment. Preliminary studies have shown that when female mice are exposed to a similar paradigm of automically inverted MF for $1 \mathrm{~h}$ at night, brainstem levels of both 5HT and 5HIAA are higher than those in unexposed control mice. If this finding is verified, then the neurochemical as well as the physiological and behavioral consequences of these field exposures may be much more widespread than originally anticipated.

\section{COMMENTARY ON THE FINDINGS AND PHYSIOLOGICAL SIGNIFICANCE}

Judging from the results reported by Lerchl and colleagues $(14,15)$, it appears that the electric transients (the eddy currents) induced by the instantaneous inversion of the horizontal component of the geomagnetic field play an important role in the observed alterations in pineal 5HT metabolism. The induced eddy currents could either directly influence pineal metabolism or the effect could be indirect via the sympathetic innervation to the organ (Fig. 1). The studies also indicate that the mere inversion of the MF, without the induction of eddy currents, is ineffective in causing a change in pineal metabolism.

There are several means by which the induced eddy currents could interact with the organism to induce changes in pineal 5HT metabolism. As noted previously, the rhythmic production of pineal melatonin is governed by the prevailing light:dark cycle, and the superimposition of light at night (like $M F$ inversion) induces a rapid reduction in pineal melatonin synthesis (16). Thus, the inverted MF could be acting like light at the level of the eyes. Indeed, Olcese et al. (11) claim that the eyes are a necessary link in the induction of pineal changes after weak static MF exposure. MF fluctuations can, in fact, produce magnetophosphenes (visual sensations initiated by magnetic fields) in the eye that may be interpreted by the retinas as light (18-20); as already mentioned, light is a well-known inhibitor of pineal melatonin production in animals and humans (16). However, it is generally conceded that much higher field strengths than those used in the studies by Lerchl et al. $(14,15)$, i.e., 0.4 Gauss, are required to induce the visual impressions of light referred to as phosphenes (21). Nevertheless, this possibility as an explanation for the observed depression in pineal $5 \mathrm{HT}$ metabolism after pulsed MF exposure cannot be completely excluded.

Other explanations have been presented to explain the bioeffects of electromagnetic field exposure on cellular physiology. Thus, the eddy currents could directly depolarize photoreceptors that would cause electrical changes in the neural pathway between the eyes and the pineal gland. That field strengths in the range used in these studies have cellular effects is not without precedence (22-24). Synaptic transmis- 


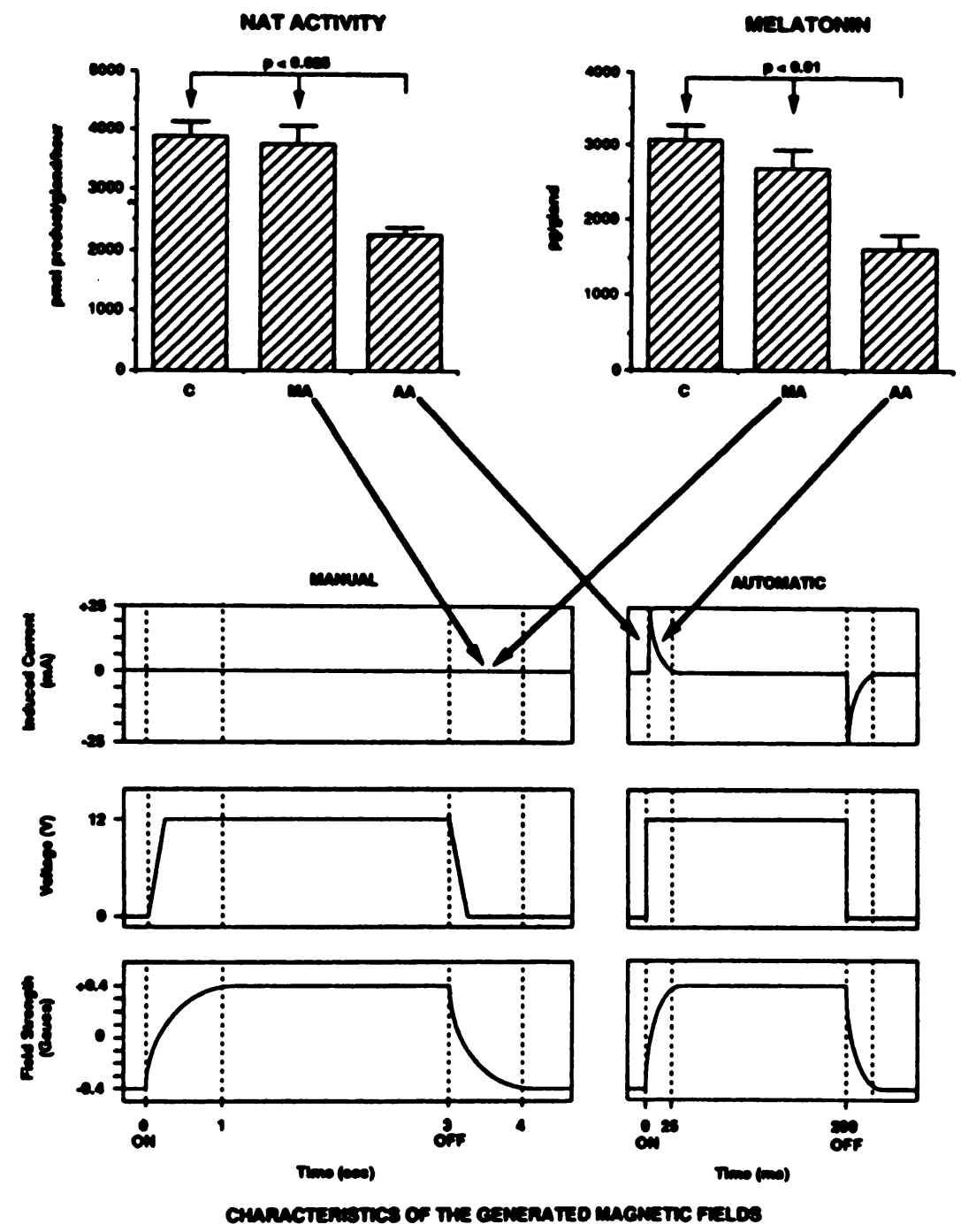

Figure 4. Induced changes in pineal NAT activity and melatonin content (top) and characteristics of the fields (bottom) used in the studies by Lerchl et al. $(14,15)$. The following parameters of the fields are shown on the bottom half of the figure: the applied voltage (middle panel) (12 volts), the resultant inversion in the MF (bottom panel), and the induced current in a search coil placed between the Helmholtz coils (upper panel). The dotted lines indicate the time period required for the inversion of the MF. When the coils were automatically activated (AA) by means of a relay switch, pineal NAT activity and melatonin levels were depressed; conversely, when the coils were activated manually (MA) by means of an integrating potentiometer over roughly a 1-s interval, no effect on pineal melatonin biosynthesis was apparent.

sion in the superior cervical ganglia (which give rise to the postganglionic fibers that innervate the pineal gland; Fig. 1) has been shown to be changed by low-strength electric fields (25). Also, it has shown that $\beta$-adrenergic receptors (which are the receptors that primarily mediate the nighttime rise in pineal melatonin production; 5,6$)$ are internalized in the membranes of cells exposed to electromagnetic fields. If the eddy currents induced by the inverted MF in the experiments of Lerchl and colleagues $(14,15)$ cause any of the changes described by the authors cited, then pineal indole-
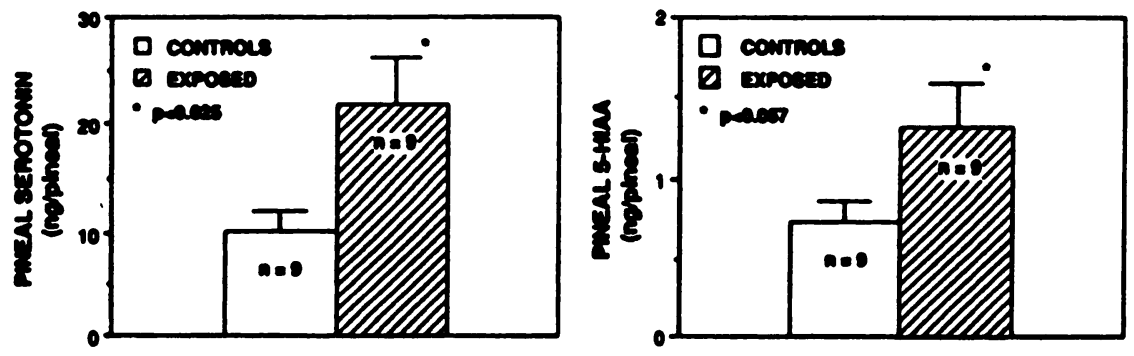

Figure 5. Pineal serotonin (5HT) and 5-hydroxyindole acetic acid (5HIAA) levels in female mice exposed to automatically and repeatedly inverted geomagnetic fields for $1 \mathrm{~h}$ during the night. The bars represent the means \pm SEM. Open bars are the values in the unexposed control animals while the cross-hatch bars are the exposed animals. 
amine metabolism could be indirectly influenced in the way observed in these experiments. Finally, the effects of the eddy currents could be directly within the pinealocytes themselves. Certainly, work from a variety of sources suggests that basic cellular processes are modified when cells are exposed to electromagnetic fields at very low energy levels $(26,27)$. Nevertheless, how pulsed, static MF exposure changes the nocturnal production of melatonin in experimental animals remains the subject of future studies. The results of Lerchl et al. $(14,15)$ indicate, however, that geomagnetic fields do not affect pineal metabolism due to their presence alone, either directly or indirectly; rather electric eddy currents produced as a result of rapid (in the low msecond range) rises and decays of the MF are a likely factor in the induction of the pineal changes.

The findings described herein are important to the field generally because they help to clarify the mechanisms whereby MF interact with the organism to alter physiology. In most studies described herein, static magnetic fields were used; however, interruption of alternating currents (e.g., 60 $\mathrm{Hz}$ ) also induces eddy currents in organisms. Thus, the present studies may also help to elucidate the mechanisms whereby these fields modify organismal physiology.

The physiologic consequences of reduced nocturnal melatonin production and secretion could be many. Melatonin is an important and widely acting hormone. Among other changes, any or all of the following may relate to a depressed circadian melatonin rhythm induced by exposure of animals to electromagnetic fields: circadian rhythm disturbances (28), fatigue and lethargy (29), mood alterations (30, 31), endocrine malfunction (32), immunodeficiency (33), and increased cancer risk (34-35). Whereas the potential increased incidence of certain types of cancer as a result of electromagnetic field exposure has received a great deal of attention (36), the other consequences may in fact be more widespread and have a greater economic and social effect.

\section{REFERENCES}

1. Gould, J. L. (1984) Magnetic field sensitivity in animals. Annu. Rev. Physiol. 46, 585-598

2. Wilson, B. W., Stevens, R. G., and Anderson, L. E. (1989) Neuroendocrine mediated effects of electromagnetic-field exposure: possible role of the pineal gland. Life Sci. 45, 1319-1332

3. Villa, M., Mustarelli, P., and Caprotti, M. (1991) Biological effects of magnetic fields. Life Sci. 49, 85-92

4. Reiter, R. J. (1981) The mammalian pineal gland: structure and function. Am. J. Anat. 162, 287-313

5. Sugden, D. (1989) Melatonin biosynthesis in the mammalian pineal gland. Experientia 45, 922-929

6. Reiter, R. J. (1991) Pineal melatonin: cell biology of its synthesis and of its physiological interactions. Endocrine Rev. 12, 151-180

7. Reiter, R. J. (1986) Normal patterns of melatonin levels in the pineal gland and body fluids of humans and experimental animals. J. Neural Transm. (Suppl.) 21, 35-54

8. Welker, H. A., Semm, P., Willig, R. P., Commentz, J. C., Wiltschko, W., and Vollrath, L. (1983) Effects of an artificial magnetic field on serotonin $\mathrm{N}$-acetyltransferase activity and melatonin content in the rat pineal gland. Exp. Brain Res. 50, 426-432

9. Semm, P., Schneider, T., and Vollrath, L. (1980) Effects of an earth-strength magnetic field and electrical activity of pineal cells. Nature (London) 288, 607-608

10. Reuss, S., Semm, P., and Vollrath, L. (1983) Different types of magnetically sensitive cells in the rat pineal gland. Neurosci. Lett. 40, 23-26

11. Olcese, J., Reuss, S., and Vollrath, L. (1985) Evidence for the involvement of the visual system in mediating magnetic field effects on pineal melatonin synthesis in the rat. Brain. Res. 333, 382-384
12. Rudolph, K., Wirz-Justice, A., Krauchli, K., and Feer, H. (1988) Static magnetic fields decrease nocturnal pineal cAMP in the rat. Brain Res. 446, 159-160

13. Stehle, J., Reuss, S., Schröder, H., Herschel, M., and Vollrath, L. (1988) Magnetic field effects on pineal N-acetyltransferase activity and melatonin content in the gerbil - role of pigmentation and sex. Physiol. Behav. 44, 91-94

14. Lerchl, A., Nonaka, K. O., Stokkan, K.-A., and Reiter, R. J. (1990) Marked rapid alterations in nocturnal pineal serotonin metabolism in mice and rats exposed to weak intermittent magnetic fields. Biochem. Biophys. Res. Commun. 169, 102-108

15. Lerchl, A., Nonaka, K. O., and Reiter, R. J. (1991) Pineal gland "magnetosensitivity" to static magnetic fields is a consequence of induced electric currents (eddy currents). J. Pineal Res. 10, 109-116

16. Reiter, R. J. (1985) Action spectra, dose-response relationships and temporal aspects of light's effects on the pineal gland. Ann. N.Y. Acad. Sci. 453, 215-231

17. Quay, W. B. (1974) Pineal Chemistry pp. 143-145, Charles C Thomas, Springfield, Ill.

18. Barlow, H. B., Kohn, H. I., and Walsh, E. G. (1947) Visual sensations aroused by magnetic fields. Am. J. Physiol. 148, 372-375

19. Oster, G. (1962) Phosphenes. Sci. Am. 222, 83-87

20. Valentinuzzi, M. (1962) Theory of magnetophosphenes. Am. J. Med. Electronics 1, 112-121

21. Lövsund, P., Nilsson, S. E. G., Reuter, T., and Öberg, P. (1980) Magnetophosphenes. A quantitative analysis of thresholds. Med. Biol. Engin. Comput. 18, 326-334

22. Adey, W. R. (1981) Tissue interactions with non-ionizing electromagnetic fields. Physiol. Rev. 61, 435-514

23. Liboff, A. R., Williams, T., Jr., Strong, D. M., and Wistar, R. J. (1984) Time-varying magnetic fields: effect on DNA synthesis. Science 223, 818-820

24. Goodman, R., Wei, L.-X., Xu, J.-C., and Henderson, A. S. (1989) Exposure of human cells to low frequency electromagnetic fields results in quantitative changes in transcripts. Biochim. Biophys. Acta 1009, 216-220

25. Jaffe, R. A., Laszweski, B. L., Carr, D. B., and Phillips, R. D. (1980) Chronic exposure to a $60-\mathrm{Hz}$ electric field: effects on synaptic transmission and peripheral nerve function in the rat. Bioelectromagnetics 1, 131-137

26. Goodman, E. M., Sharpe, P. T., Greenebaum, B., and Marron, M. T. (1986) Pulsed magnetic fields alter the cell surface. FEBS Lett. 199, 275-278

27. Byus, C. V., Reiper, S. E., and Adey, W. R. (1987) The effects of low-energy 60 - $\mathrm{Hz}$ environmental electromagnetic fields upon the growth-related enzyme ornithine decarboxylase. Carcinogenesis 8, 1385-1389

28. Armstrong, S. M. (1989) Melatonin and circadian control in mammals. Experientia 45, 932-938

29. Arendt, J., Aldhous, M., English, J., Marks, V., Arendt, J. H., Marks, M., and Folkard, S. (1987) Some effects of jet lag and their alleviation by melatonin. Ergonomics 30, 1379-1393

30. Stanley, M., and Brown, G. M. (1988) Melatonin levels are reduced in the pineal glands of suicide victims. Psychopharmacol. Bull. 24, 484-488

31. Arendt, J. (1989) Melatonin: a new probe in psychiatric investigation? Br. J. Psychiatr. 155, 585-590

32. Reiter, R. J. (1986) Pineal function in the human: implications for reproductive biology. J. Obstet. Gynecol. 6, S77-S81

33. Maestroni, G. J. M., Conti, A., and Pierpaoli, W. (1988) Pineal melatonin, its fundamental immunomodulatory role in aging and cancer. Ann. N.Y. Acad. Sci. 521, 140-148

34. Tamarkin, L., Danforth, D., Lichter, A., Demoss, E., Cohen, M., Chabner, B., and Lippman, M. (1982) Decreased nocturnal plasma melatonin peak in patients with estrogen receptor positive breast cancer. Science 216, 1003-1005

35. Wilson, B. W., Lueng, F., Buschbom, R., Stevens, R. G., Anderson, L. E., and Reiter, R. J. (1988) Electric fields, the pineal gland and cancer. In The Pineal Gland and Cancer (Gupta, D., Attanasio, A., Reiter, R. J., eds) pp. 245-259, Brain Research Promotion, Tübingen, Germany

36. Phillips, R. G. (1987) Electric power use and breast cancer: a hypothesis. Am. J. Epidemiol. 125, 556-561 